\section{Leave it to cleaver}

A new strategy for the modulation of ribozyme-mediated RNA cleavage may provide investigators with a powerful system for transgene regulation that bypasses the complications associated with conventional transcriptional control methods.

Numerous highly efficient self-cleaving catalytic RNAs, or 'ribozymes,' have been identified in nature over the past two decades. These ribozymes promised untapped potential to Harvard researcher Richard Mulligan, a gene transfer specialist interested in developing simple, safe approaches to the regulation of exogenous genes that would eliminate the need for multiple synthetic constructs and chimeric transactivators. "We immediately considered mechanisms other than regulation at the level of transcription," he says, and ribozymes seemed a logical choice. Theoretically, when constructs are used that directly incorporate ribozymes within a given transcript, no expression should occur unless cleavage is blocked (Fig. 1).

Mulligan was initially encouraged when an examination of the research literature revealed numerous evidence that suggested- "I emphasize suggested," he adds pointedly-the availability of numerous ribozymes capable of efficient cleavage in vitro, many of which could be readily inhibited by a variety of antibiotic compounds. Unfortunately, this in vitro promise did not yield in vivo payoffs, and virtually none of the more than 100 ribozymes that they screened demonstrated significant inhibitory properties in mammalian cells.

Mulligan cites lead author Laising Yen's steadfastness as a key factor in their eventual success: "I was lucky I had a very able postdoc, because it took years to make this happen." In the end, they identified two ribozymes capable of reasonable cleavage in mammalian cells; however, even the stronger of the two, Sm1, was relatively inefficient, and the researchers set about further optimizing the ribozyme.

After a series of modifications to the Sm1 sequence, the team developed N79, a clone that triggered highly efficient cleavage and subsequent degradation of reporter transcripts when positioned immediately $5^{\prime}$ of the reporter gene coding sequence, resulting in 225 -fold inhibition of expression. However, their enthusiasm was dampened by the discovery that none of the antibiotics they tested showed the inhibitory properties demonstrated in vitro.

This led to another exhaustive round of experiments: high-throughput chemical screens to identify drugs that promote the appropriate level of transgene activation, using mammalian cells transfected with an N79-controlled luciferase construct. The nucleoside analogue toyocamycin proved to be the most effective compound, with treated cells showing a marked increase in luciferase expression.

Mulligan's team then put their optimized ribozyme-inhibitor pair to the true test, using an adeno-associated viral construct to introduce N79-regulated luciferase into the retinas of mice. Three weeks after injection, some of the mice were implanted with pellets for the gradual release of toyocamycin; 48 hours after implantation, lucifer-

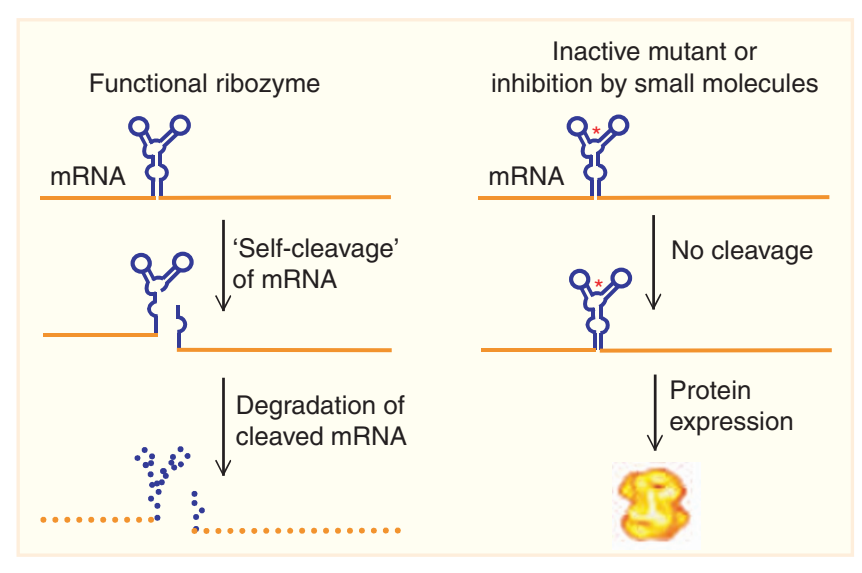

Figure 1 | An overview of the Mulligan group's strategy for ribozyme-based regulation of gene expression. Reprinted with permission from Nature.

ase expression was considerably elevated (between 39- and 191-fold) in the retinas of treated mice, but absent in untreated controls.

Yale researcher Ron Breaker knows a thing or two about catalytic RNAs, having dedicated much of his career to their study and described this approach as "a tour-de-force engineering effort". He adds, "I think the big breakthrough—at least the first big breakthrough - is that they actually got a ribozyme slotted into a messenger RNA to work. I think this is a major achievement... it has been very difficult to just simply drop these ribozymes into messenger RNAs and get them to knock down gene expression." His one concern is that better inhibitors will be needed, as toxicity is a problem with toyocamycin.

Mulligan recognizes this concern: "I would emphasize that this is a proof-of-principle report... these are not ideal compounds. But probably the most important aspect of this for the future is that, in contrast to the existing gene regulation systems, the way this system is set up makes it highly likely that we will be able to now generate gene transfer systems tailored to any small molecule." To better fulfill this system's promise, Mulligan's and Breaker's labs are collaborating to engineer and characterize ribozyme variants capable of exquisite regulation in response to a ligand of choice, and both researchers anticipate that with a little more work, this system has the potential to offer unprecedented capabilities for regulated gene transfer in the near future.

\section{Michael Eisenstein}

\section{RESEARCH PAPERS}

Yen, L. et al. Exogenous control of mammalian gene expression through modulation of RNA self-cleavage. Nature 431, 471-476 (2004). 